\title{
From Discrete Hopping to Continuum Modeling on Vicinal Surfaces with Applications to $\mathrm{Si}(001)$ Electromigration
}

\author{
Tong Zhao ${ }^{1}$ and John D. Weeks ${ }^{2}$ \\ ${ }^{1,2}$ Institute for Physical Science and Technology, \\ University of Maryland, College Park, Maryland 20742 and \\ ${ }^{2}$ Department of Chemistry and Biochemistry, University of Maryland, College Park, Maryland 20742 \\ Daniel Kandel ${ }^{3}$ \\ ${ }^{3}$ Department of Physics of Complex System, Weizmann Institute of Science, Rehovot 76100, Israe*
}

(Dated: June 28, 2018)

\begin{abstract}
Coarse-grained modeling of dynamics on vicinal surfaces concentrates on the diffusion of adatoms on terraces with boundary conditions at sharp steps, as first studied by Burton, Cabrera and Frank (BCF). Recent electromigration experiments on vicinal Si surfaces suggest the need for more general boundary conditions in a BCF approach. We study a discrete 1D hopping model that takes into account asymmetry in the hopping rates in the region around a step and the finite probability of incorporation into the solid at the step site. By expanding the continuous concentration field in a Taylor series evaluated at discrete sites near the step, we relate the kinetic coefficients and permeability rate in general sharp step models to the physically suggestive parameters of the hopping models. In particular we find that both the kinetic coefficients and permeability rate can be negative when diffusion is faster near the step than on terraces. These ideas are used to provide an understanding of recent electromigration experiment on $\mathrm{Si}(001)$ surfaces where step bunching is induced by an electric field directed at various angles to the steps.
\end{abstract}

\section{INTRODUCTION}

Steps on vicinal crystal surfaces, created by a miscut along a low index plane, have long been of great interest in both basic and applied research 1 High quality crystals can be grown through step-flow - the uniform motion of more or less equally-spaced steps — and step bunching instabilities can create arrays of wide flat terrace separated by closely bunched steps. Other arrangements of steps could serve as templates for nanoscale structures and devices.

Most fundamental studies of the static and dynamic properties of vicinal surfaces are based on generalizations of the classic theory of Burton, Cabrera, and Frank $(\mathrm{BCF})$, developed more than fifty years ago 2 This theory describes the diffusion of adatoms on terraces with boundary conditions at steps, which are treated as sharp line boundaries. Originally BCF assumed that the steps acted as perfect sinks and sources of adatoms so that the limiting adatom concentration at the step boundaries always reduces to local equilibrium.

Many extensions and modifications of the BCF theory have been suggested to provide a more general framework for the description of different experiments. One of the most important was Chernov's introduction of linear kinetic coefficients, which permit deviations from local equilibrium at steps ${ }^{3.4}$ It was soon recognized that in general the kinetic coefficients could be asymmetric. $\frac{5}{-}$ Another generalization permits step permeability or transparency, with a term in the boundary condition directly connecting the limiting adatom concentration on adjacent terraces. $\frac{6}{-}$ These generalized BCF models provide a mesoscopic or coarse-grained description of surface evolution with effective boundary conditions at sharp steps, and we will generally refer to them as sharp step models.

Many kinetic instabilities seen in experiments have been successfully described from this perspective using various combinations of boundary conditions. However in general it is not clear how to connect the choices and values of the effective parameters in sharp step models to the underlying physical processes or how to determine the uniqueness of such a mapping. A similar difficulty arises in trying to relate "microscopic" parameters in kinetic Monte Carlo simulations of discrete hopping models to the effective parameters in a generalized sharp step model. Very different microscopic models can sometimes seem to give equally plausible mesoscopic descriptions of limited sets of experimental data.

In previous work ${ }^{7}$ we proposed a novel continuum tworegion diffusion model (CTRM), which gave a rather simple and unified description of a variety of current-induced instabilities seen experimentally on vicinal Si surfaces. We will discuss these experiments in more detail later. The model assumes that diffusion rates in a finite region around a step could be affected by the different local bonding configurations and thus differ from those found elsewhere on terraces. By extrapolating the steady state concentration profiles to the center of the step region, we obtained a mapping of the parameters in the CTRM to those of an equivalent classical sharp step model. One surprising conclusion was that negative kinetic coefficient can arise when the diffusion rate near a step is faster than that on the terraces.

In this paper, we will provide a more systematic way of deriving the boundary conditions for the continuum sharp step models from a rather general 1D discrete hopping model that permits both asymmetric diffusion in the step region as well as step permeability. As discussed by 


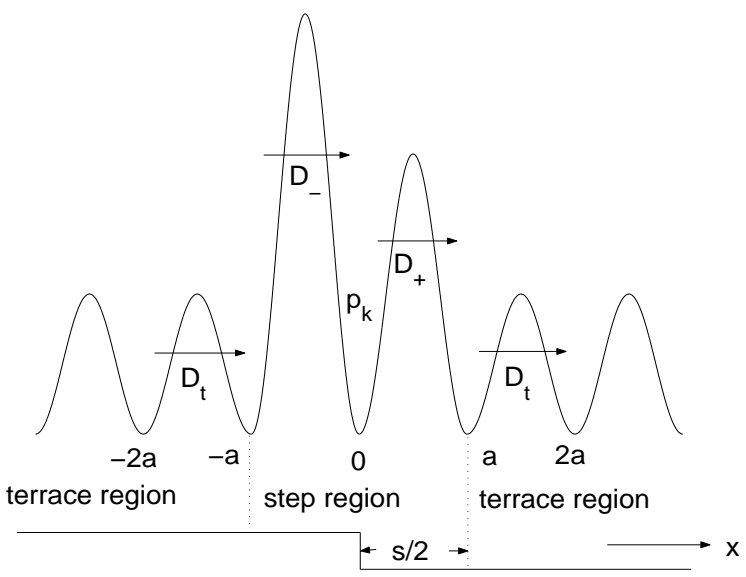

FIG. 1: A schematic plot of the 1D potential surface near an atomic step. Different $D$ 's that have dimensions of diffusion constants characterize the hopping rates associated with different barrier heights. Here, we take the width of the step region to be $2 a$.

Ghez and Iyer,,$\frac{8}{,}$ such an effective $1 \mathrm{D}$ model can result from averaging over relevant $2 \mathrm{D}$ configurations of kink and ledge sites on an atomic step. We then use these ideas to provide a detailed description of electromigration on $\mathrm{Si}(001)$ surfaces, and find a coherent scenario that explains most of the interesting experimental findings.

\section{1D HOPPING MODEL}

The simple 1D model that we study is schematically shown in Fig. 11 where an atomic step site is surrounded by a region of width $s$ with generally different diffusion rates, induced by reconstruction or rearrangements of local dangling bonds. As we will see, this difference can generate effective kinetic coefficients in a sharp step description.

In general, the width $s$ of the step region with different diffusion barriers should vary for different systems. However, we find that the essential physics of the hopping model is not strongly affected by specific choices of $s$ of order of a few lattice spacings $a$. Thus we analyze the algebraically simplest case shown in Fig. 1 with half-step regions of width $a$. The more general result, needed in the analysis of $\mathrm{Si}(001)$ experiments, is easily obtained by replacing $a$ with $s / 2$, as will become clear later when we compare the results of this generic hopping model to our previous results.

We include here two additional physical features of the step region as illustrated in Fig. [1 One is the possible asymmetry in the diffusion processes in the up and down half-step regions, described by hopping rates $D_{ \pm} / a$. The $D_{ \pm}$have dimensions of a diffusion constant, and the model is usefully characterized by dimensionless parameters

$$
R_{ \pm} \equiv D_{ \pm} / D_{t}
$$

with $D_{t}$ the diffusion constant on the terraces.

The other feature we build in is step permeability or transparency, characterized in our model by a single parameter $p_{k}\left(0 \leq p_{k} \leq 1\right)$. This can be understood as the effective probability in our 1D model that an adatom hopping to site 0 will encounter a kink site at a given temperature and thus equilibrate with the solid. This parameter takes account of effects from both kink site density and ledge diffusion in a full 2D model. When $p_{k}=1$, the step site acts as a perfect sink maintained by either enough kink sites or fast ledge diffusion or both, and consequently the step site concentration will be pinned at equilibrium $c_{e q}$. In the opposite limit with $p_{k}=0$ no adatoms are incorporated into the solid. The step site behaves like any other terrace site and thus is perfectly permeable. We neglect other possible sources of permeability, including direct hopping over the step region from one terrace to another or effects of rapid step motion, 9 which we believe are less physically relevant for our cases of interest. In this section we will also consider only diffusion fluxes from concentration gradients, and discuss the case of driven diffusion from an external field in the Appendix.

We assume that the net flux of adatoms that hop between step site 0 and site $a$ can be partitioned into two effective contributions:

$$
J_{a / 2}=\frac{D_{+}}{a}\left[p_{k}\left\{c_{e q}-\hat{c}(a)\right\}+\left(1-p_{k}\right)\{\hat{c}(0)-\hat{c}(a)\}\right] .
$$

The first term describes an adatom exchange with probability $p_{k}$ involving equilibrated "kink-like" adatoms at site 0 with density $c_{e q}$ and the neighboring terrace site. The second term involves a similar exchange with probability $\left(1-p_{k}\right)$ involving unincorporated "ledge-like" adatoms with density $\hat{c}(0)$. Only the former involves creation/annihilation of adatoms, and the latter is treated as a normal diffusion flux that conserves the adatom density.

Similarly, the flux from site $-a$ to 0 is

$J_{-a / 2}=\frac{D_{-}}{a}\left[p_{k}\left\{\hat{c}(-a)-c_{e q}\right\}+\left(1-p_{k}\right)\{\hat{c}(-a)-\hat{c}(0)\}\right]$.

Since we assume that all the sinks/sources reside only at site 0 , the net flux of adatoms that hop from site $a(-2 a)$ to site $2 a(-a)$ takes on the simpler form

$$
J_{ \pm 3 a / 2}= \pm \frac{D_{t}}{a}[\hat{c}( \pm a)-\hat{c}( \pm 2 a)] .
$$

As in many other situations of physical interest, we will use the quasi-static approximation to simplify the analysis. Here we assume that the motion of the step region is much slower than the relaxation of the terrace diffusion field, so that one can determine the diffusion process on terrace sites with fixed positions of the step regions. In the quasi-static limit the net change in the number of adatoms at each terrace site given by a total flux balance must vanish, i.e., $d \hat{c}(x) / d t=0$ for all $x= \pm a, \pm 2 a \ldots$ In 
particular, at sites $\pm a$, the balance of fluxes is given by

$$
0=\frac{d \hat{c}( \pm a)}{d t}= \pm\left[a J_{ \pm a / 2}-a J_{ \pm 3 a / 2}\right] .
$$

At step site $0, \hat{c}(0)$ can be determined by balancing the conserved flux terms proportional to $\left(1-p_{k}\right)$ in Eqs. (2) and (31), and is given by

$$
\hat{c}(0)=\frac{D_{+} \hat{c}(a)+D_{-} \hat{c}(-a)}{D_{+}+D_{-}} .
$$

\section{RELATING PARAMETERS IN DISCRETE AND CONTINUUM MODELS}

Our task now is to relate the physically suggestive parameters $R_{ \pm}$and $p_{k}$ in the discrete hopping model to the kinetic coefficients $k_{ \pm}$and permeability rate $P$ appearing in the boundary conditions of a continuum sharp step model as in Eq. (77) below. For $x>0$, consider a smooth continuum concentration profile $c(x)$ that passes through the discrete concentrations $\hat{c}(a)$ and $\hat{c}(2 a)$. (The caret distinguishes discrete from continuum functions.) The behavior of $c(x)$ at larger $x$ is determined by the physical processes on the terraces, but does not need to be specified explicitly for our purposes here.

To make contact with the sharp step model, we rewrite the fluxes in Eqs. (2)-(5) in terms of $c(x)$. To that end we use a Taylor series expansion to linear order to express $c(a)=\hat{c}(a)$ and $c(2 a)=\hat{c}(2 a)$ in terms of $c^{+} \equiv c\left(0^{+}\right)$, the extrapolated limiting concentration as $x \rightarrow 0^{+}$at the sharp step edge in a continuum picture, and its associated gradient $\left.\nabla c\right|_{+}$. Similarly, $\hat{c}(-a)$ and $\hat{c}(-2 a)$ can be expressed in terms of $c^{-}$and $\left.\nabla c\right|_{-}$, which in general are different than $c^{+}$and $\left.\nabla c\right|_{+}$.

Using Eq. (6) to eliminate $\hat{c}(0)$, and substituting into Eq. (5), we find that the result can be rewritten in the form of a generalized linear kinetics boundary condition ${ }^{8}$ with permeability

$$
\pm\left[\left.D_{t} \nabla c\right|_{ \pm} \mp v c^{ \pm}\right]=k_{ \pm}\left(c^{ \pm}-c_{e q}\right)+P\left(c^{ \pm}-c^{\mp}\right) .
$$

The kinetic coefficients $k_{ \pm}$are given by

$$
k_{ \pm}=\frac{D_{t}}{a} \frac{p_{k}}{\left(R_{ \pm}-1\right)\left[1+\left(1-p_{k}\right) M\right]},
$$

where

$$
M \equiv \frac{R_{+} R_{-}}{\left(R_{+}+R_{-}\right)}\left[\frac{R_{+}}{R_{-}-1}+\frac{R_{-}}{R_{+}-1}\right]
$$

is symmetric on exchange of + and - . Note in general that the ratio of the kinetic coefficients satisfies

$$
\frac{k_{+}}{k_{-}}=\frac{R_{-}-1}{R_{+}-1}
$$

independent of $p_{k}$. The permeability rate $P$ can be written as

$$
P=\frac{k_{ \pm}}{p_{k}\left(R_{\mp}-1\right)} \cdot \frac{\left(1-p_{k}\right) R_{+} R_{-}}{\left(R_{+}+R_{-}\right)} .
$$

Using Eq. (8) in the first factor, we see that $P$ is symmetric on exchange of + and - , and has a finite limit as $p_{k} \rightarrow 0$.

The final parameter $v$ in Eq. (7) is zero in our present treatment since we used the quasi-static approximation to derive Eqs. (5) and (6). In principle, a non-vanishing $v$ would arise if we took the flux due to step motion into account in the discrete hopping model. However, the quasi-static limit is valid in most physical cases of interest, and thus this additional complication can be avoided.

Equations (711) are the central results in this section. As mentioned earlier, we find that the sharp step boundary condition can indeed be generally expressed using linear kinetics with permeability. More importantly, we are able to relate the effective parameters in the sharp step boundary conditions to the physically suggestive parameters we considered in our generic hopping model. This mapping provides a simple way to understand many aspects of electromigration phenomena on Si surfaces.

A notable general feature of these equations is that the kinetic coefficients $k_{ \pm}$are proportional to $p_{k}$ and the permeability rate $P$ is proportional to $\left(1-p_{k}\right)$. The kinetic coefficients characterize adatom exchange involving equilibrated solid adatoms at kinks and the adatom gas phase, while the permeability rate characterizes adatom motion across the step without equilibrating with the solid phase. Moreover, the kinetic coefficients $k_{ \pm}$are in general asymmetric on the two sides of the step due to the asymmetry of emission and diffusion processes from kinks. However, the permeability rate $P$ is symmetric since the physical processes of hopping from one side to the other without attachment at the step always involves the diffusion constants on both sides.

We now consider some limits of the above general expressions to illustrate some interesting features of both the kinetic coefficients and the permeability rate.

\section{A. Impermeable steps, $p_{k} \rightarrow 1$}

This limit is usually considered in treatments of the sharp step model, and we used it to analyze currentinduced instabilities on Si surfaces ${ }^{7}$ In this limit the only way for the adatoms to go across a step is through attachment/detachment at kinks, and the permeability rate $P$ vanishes.

The results are conveniently described in terms of the attachment/detachment lengths

$$
d_{ \pm} \equiv D_{t} / k_{ \pm}
$$

Using Eq. (8), these are given by

$$
d_{ \pm}=a\left(R_{ \pm}-1\right) .
$$

If we replace the width $2 a$ of the step region in the present model by a general value $s$, we recover exactly the results we found earlier ${ }^{7}$ using the CTRM. As shown in the appendix, Eq. (13) also holds to lowest order even when 
there is an external driving field that affects processes in the step region.

This shows the general validity of the mapping between model parameters that we found earlier by considering diffusion driven by a weak electric field. For $R_{ \pm}>1$, corresponding to slower diffusion in the step region, the attachment/detachment lengths and kinetic coefficients are positive. The kinetics is usually called attachment/detachment limited when $d_{ \pm} \gg l$, with $l$ the average terrace width in a uniform step train, or diffusion limited when $0 \leq d_{ \pm} \ll l$. For $R_{ \pm}=1, d_{ \pm}$vanishes, and the kinetic coefficients diverge. This forces $c^{ \pm}$to equal $c_{e q}$ in Eq. (7) and generates the local equilibrium boundary condition originally proposed in the BCF model.

More interestingly, for $R_{ \pm}<1$, corresponding to faster diffusion in the step region, the attachment/detachment lengths and the corresponding kinetic coefficients are $n e g$ ative. As we showed earlier, 7 the sign of the kinetic coefficients plays a key role in interpreting electromigration experiments on Si surfaces, since it determines the stability of a uniform step train for a given current direction. This application of our general results will be discussed in more detail below.

In the following, we will characterize the limit $p_{k} \rightarrow 1$ as defining a perfect sink model, since adatoms can not diffuse across a step without attachment/detachment at kink sites. As a direct consequence, the two sides of the step are decoupled and any change of the microscopic rates on one side of the step does not affect the kinetic coefficient on the other side. However, as shown above, the two sides of the step will in general be coupled for $p_{k}<1$ through Eqs. (10) and (11), and the subsequent analysis of step dynamics becomes much more involved.

\section{B. Very permeable steps, $p_{k} \rightarrow 0$}

This limit may be physically relevant at low enough temperatures, or slow enough ledge diffusion, or some proper combination of both. Here the adatoms hop around on the surface without encountering sinks/sources in the step region. Thus one expects vanishing kinetic coefficients, but a finite permeability rate, and this is indeed what Eq. (8) and Eq. (11) predict in this limit.

As in Eq. (12), let us define a corresponding permeability length

$$
d_{P} \equiv D_{t} / P
$$

Then Eqs. (11) and (8) yield

$$
d_{P}=2 a\left[\frac{1}{2}\left(R_{+}+R_{-}\right)-1\right] .
$$

Similar to $d_{ \pm}$, the permeability length $d_{P}$ can become negative when $\left(R_{+}+R_{-}\right)<2$, with faster diffusion in the step region. Eq. (15) is consistent with results derived from a continuum phase field model ${ }^{\frac{9}{*}}$ Recently

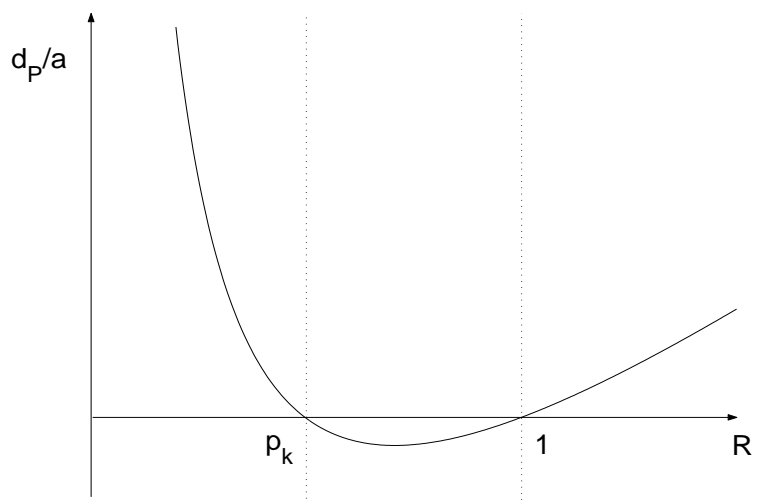

FIG. 2: Plot of the dimensionless permeability length $d_{P} / a$ as a function of $R$ in the symmetric case for a general $p_{k}$.

Pierre-Louis and Métois ${ }^{10}$ have argued that negative permeability lengths can explain some novel growth-induced instabilities seen during electromigration on $\mathrm{Si}(111)$ surfaces.

\section{Partially permeable steps, $0<p_{k}<1$}

This is the most general case, where only a finite fraction of adatoms at the step equilibrate at kinks, presumably corresponding to intermediate temperatures with moderate ledge diffusion. We focus on the simplest symmetric case where $D_{ \pm}=D_{s}$ or $R_{+}=R_{-}=R$ in Eqs. (8)-(11). The attachment/detachment length becomes

$$
d=\frac{a}{p_{k}}\left(R-p_{k}\right)
$$

and the permeability length is

$$
d_{P}=2 a(R-1) \frac{\left(R-p_{k}\right)}{\left(1-p_{k}\right) R} .
$$

Equation (16) can be understood using the same physics as in the perfect sink model. With a finite probability $p_{k}$ to encounter a kink, an adatom has to move faster in the step region $\left(D_{s}=D_{t} / p_{k}\right)$ to maintain local equilibrium $(d \rightarrow 0$ or $k \rightarrow \infty)$ compared with the perfect sink case $\left(D_{t}=D_{s}\right)$. The permeability length in Eq. (17) is a new feature arising from the possibility that the adatoms go directly across the step without equilibrating with the solid. This expression shows a fairly complicated dependence on microscopic motions characterized by $R$ and $p_{k}$.

A schematic plot of $d_{P}$ versus $R$ for a given $p_{k}$ is shown in Fig. 2] Both $d$ and $d_{P}$ diverge as $R \rightarrow \infty$, since all motion in the step region vanishes in this limit. $d_{P}$ decreases as $R$ decreases, and stays positive for $R>1$. Just like the attachment/detachment length, the permeability length changes sign from positive to negative as $R$ passes through 1 , with equal hopping rates in the terrace and step regions. However, the permeability length becomes 
positive again for small enough $R$ when the motion in the step region is sufficiently fast $\left(R<p_{k}\right)$ that the probability of crossing the step without involvement of a kink is effectively decreased to a point that it is no longer faster than hopping on terraces.

\section{APPLICATIONS TO ELECTROMIGRATION ON SI(001)}

We now apply these ideas to current-induced instabilities on vicinal Si surfaces ${ }^{11}$ Step bunching is seen on $\mathrm{Si}(111)$ surfaces when the electric current is properly directed normal to the step direction ${ }^{12}$ The uniform step train is initially stable when the current flows in the opposite direction. There are three temperature ranges between about $850^{\circ} \mathrm{C}$ and $1300^{\circ} \mathrm{C}$ where the stable and unstable directions are reversed. However, at similar temperatures vicinal $\mathrm{Si}(001)$ surfaces miscut along [110] exhibit step bunching from current normal to the steps in both directions. ${ }^{14.15}$ Characteristic bunching patterns have also been observed for current directed at various angles to the steps, 16

There is general agreement that in the presence of an electric field adatoms acquire an effective charge $z^{*} e$ (which includes both electrostatic and a "wind-force" contribution arising from scattering of charge carriers), and thus experience a field-directed force $\mathbf{F}=z^{*} e \mathbf{E}$ that biases their diffusive motion. However, it is less clear what are appropriate boundary conditions in a sharp step model for these processes and how they might be affected by the electric field and by surface reconstruction. The generic hopping model studied in Sec. II helps us shed some light on these issues, and the results can be applied to electromigration on both $\mathrm{Si}(111)$ and $\mathrm{Si}(001)$ surfaces. In this paper we discuss applications to $\mathrm{Si}(001)$ surfaces. The different $\mathrm{Si}(111)$ instabilities will be discussed elsewhere 17

The most notable differences in current-induced step bunching on $\mathrm{Si}(001)$ and $\mathrm{Si}(111)$ surfaces arise from the $(2 \times 1)$ surface reconstruction (dimerization) on $\mathrm{Si}(001)$, which persists up to temperatures of at least $1200^{\circ} \mathrm{C}$ Two characteristic directions on the surface are established by dimerization, either parallel or perpendicular to the substrate dimer rows in the orthogonal [110] direction, denoted by $\|$ and $\perp$ respectively. Experimental evidence suggests that the diffusion along the dimer rows is much faster at low temperatures, i.e., $D_{t}^{\|} \gg D_{t}^{\perp}, 19$

In recent experiments, ${ }^{16}$ the bunching behavior was studied on dimple geometries, where steps of all orientations are found. As schematically shown in Fig. 37, there are in general two angles needed to describe the local geometry of the dimple when the electric field is applied, ${ }^{16}$ characterized by the angle $\theta$ between direction of the electric field and the [110] direction, and the angle $\varphi$ between the field and the local normal to the steps.

The bunching exhibits interesting angular dependences. When the current is parallel to the orthogonal
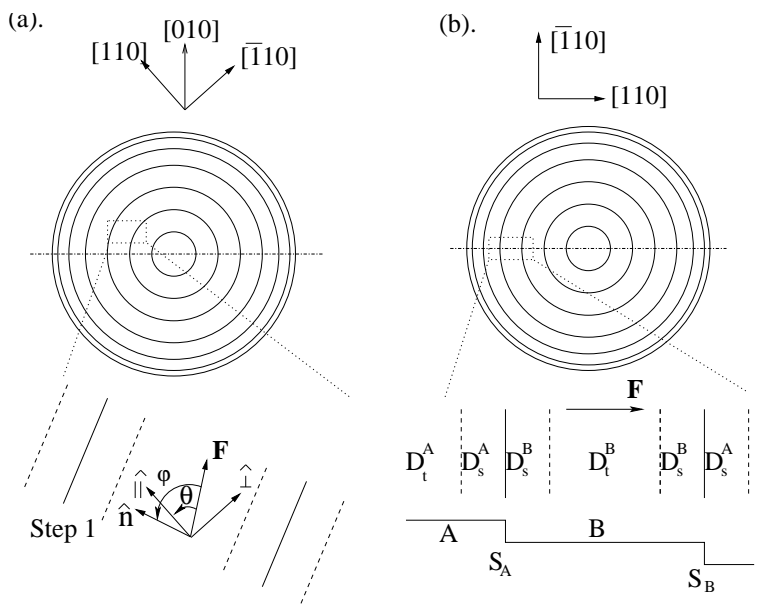

FIG. 3: A schematic illustration of the dimple geometry on the $\mathrm{Si}(001)$ surface. (a) The general view of the dimple with the crystallographic directions indicated above. Zooming into a given local area of the dimple (the dotted line box), we show the step-terrace configuration with a general direction of the electric field. $\varphi$ is the angle between field direction and the local normal to the steps, while $\theta$ is the angle between the field and [110]. $\theta=\pi / 4$ corresponds to a field direction along [010]. (b) The top view of the dimple when $\theta=0$. Zooming into the dotted-lined box near the center of the dimple with $\varphi=0$, we show a top view of the vicinal surface and a side view of the step-terrace configuration. Most of basic physics of step pairing and bunching will be illustrated in this simple $1 \mathrm{D}$ geometry with the electric field perpendicular to average step position.

[110] direction $(\theta=0)$, the bunching is observed to be strongest in the areas where the current is locally parallel to the step normal direction $(\varphi=0)$, e.g., the dotted line box in Fig. 3b. No bunching occurs in the corresponding perpendicular directions $(\varphi=\pi / 2)$. However, if the current is rotated to $\pi / 4$ off the dimer row direction $(\theta=\pi / 4)$, the strongest bunching occurs in the areas where the current is perpendicular to the local step directions $(\varphi=\pi / 2)$. No bunching is seen in the corresponding perpendicular direction $(\varphi=0)$, which in the previous case was where the maximum bunching was found. In the following discussion, we will first study the instabilities for the simplest case as shown in the dotted line box in Fig. 3] $(\theta=0$ and $\varphi=0)$, and then generalize our results to arbitrary $\theta$ and $\varphi$.

\section{A. Domain Conversion and Step Pairing}

Let us begin with the simplest case, where the vicinal surface is misoriented in the [110] direction. At equilibrium rather straight $S_{A}$ steps that run parallel to the dimer rows of the upper $A$ terrace alternate with much rougher $S_{B}$ steps that run perpendicular to the dimer rows of the upper $B$ terrace ${ }^{20}$ When the field is normal to the steps, as illustrated in the boxed region of Fig. 3b, the terrace diffusion rates normal to the steps 
satisfy $D_{t}^{B} \gg D_{t}^{A}$. We assume that the dimerization persists at least to some extent on both adjacent halfstep regions around each terrace and will similarly affect diffusion rates there. The normal diffusion in the two half-step regions around a given step is characterized by $D_{s}^{A}$ and $D_{s}^{B}$. Taking account of the differences in terrace diffusion rates, it seems reasonable to assume at least that $D_{s}^{B} \geq D_{s}^{A}$, or

$$
\left(D_{t}^{B}-D_{t}^{A}\right)\left(D_{s}^{B}-D_{s}^{A}\right) \geq 0 .
$$

Special cases of this assumption include classical local equilibrium steps where $R^{A}=R^{B}=1$ and a symmetric step model where $D_{s}^{B}=D_{s}^{A}$.

The assumption here essentially states that the fundamental physics on $\mathrm{Si}(001)$ surfaces is dominated by the alternating reconstruction domains on terraces. Under this assumption, it is natural to think of the surface as made up of alternating $A$ and $B$ units, where the unit $\alpha(\alpha=A$ or $B)$ contains an $\alpha$ terrace together with the two neighboring $\alpha$ half step regions.

We consider here cases where the system is driven away from equilibrium only by the electric field. We make the usual assumption that permeability does not play an essential role in this case and take the limit $p_{k} \rightarrow 1$ for simplicity. We also neglect evaporation and assume a positive effective charge. The perfect sink limit decouples the concentration fields on the terraces, and permits a simple solution to the steady state diffusion problem in terms of exponential functions $e^{f x}$, where $f \equiv \mathbf{F} \cdot \hat{x} / k_{B} T$.

In almost all cases of experimental interest, the field is sufficiently weak that $f s \ll f l_{t} \ll 1$, where $s$ and $l_{t}$ are the width of the step and terrace regions, and we obtain piecewise linear profiles for the adatom concentration. It is then straightforward to write down the general solution for the adatom density in unit $\alpha$ as

$$
c^{\alpha}(x)=\left\{\begin{array}{lc}
c_{e q}+m_{s}^{\alpha}\left(x+\frac{l_{t}^{\alpha}+s}{2}\right) & -\frac{l_{t}^{\alpha}+s}{2} \leq x \leq-\frac{l_{t}^{\alpha}}{2} \\
c_{e q}+m_{t}^{\alpha} x & -\frac{l_{t}^{\alpha}}{2} \leq x \leq \frac{l_{t}^{\alpha}}{2} \\
c_{e q}+m_{s}^{\alpha}\left(x-\frac{l_{t}^{\alpha}+s}{2}\right) & \frac{l_{t}^{\alpha}}{2} \leq x \leq \frac{l_{t}^{\alpha}+s}{2}
\end{array}\right.
$$

where $l_{t}^{\alpha}$ is the $\alpha$ terrace width. In the above expression the origin is set at the center of the terrace region to take maximum advantage of symmetry. It is easy to transform the origin to the left atomic step position in accordance with the previous discussion on hopping models, and the results below will not be altered by any specific choice of the coordinate system.

The $m_{s, t}^{\alpha}$ can be obtained by requiring continuity of concentration and flux at $\pm l_{t}^{\alpha} / 2$ and are given by

$$
\begin{aligned}
& m_{t}^{\alpha}\left(l_{t}^{\alpha}\right)=c_{e q} s f\left(R^{\alpha}-1\right) /\left(l_{t}^{\alpha}+R^{\alpha} s\right) \\
& m_{s}^{\alpha}\left(l_{t}^{\alpha}\right)=c_{e q} l_{t}^{\alpha} f\left(1-R^{\alpha}\right) /\left(l_{t}^{\alpha}+R^{\alpha} s\right)
\end{aligned}
$$

Here $R^{\alpha} \equiv D_{t}^{\alpha} / D_{s}^{\alpha}$ gives a dimensionless measure of the relative diffusion rates in the $\alpha$ unit between the terrace and step regions in a direction perpendicular to the step direction, and $c_{e q}$ is the average concentration for a uniform step array when $f=0$.

In the quasi-static approximation the step velocities are computed by a flux balance. The surface flux normal to the step is constant throughout the $\alpha$ unit and is exactly given by

$$
J_{0}^{\alpha}=D_{t}^{\alpha} c_{e q} f \frac{l_{t}^{\alpha}+s}{l_{t}^{\alpha}+R^{\alpha} s}
$$

Because of the perfect sink assumption, the fluxes in the individual $\alpha$ units on either side of a step are independent of each other. Thus the step velocity is easy to compute for a given step configuration.

Consider in particular the initial velocity of step $S_{\alpha}$ in a uniform step train $\left(l_{t}^{A}=l_{t}^{B}=l_{t}\right)$. This is given by

$$
\begin{aligned}
v_{0}^{\alpha} & =\Omega\left(J_{0}^{\alpha}-J_{0}^{\beta}\right) \\
& =\Omega c_{e q} f\left(l_{t}+s\right) \frac{\left[\left(D_{t}^{\alpha}-D_{t}^{\beta}\right) l_{t}+\left(D_{s}^{\alpha}-D_{s}^{\beta}\right) s R^{\alpha} R^{\beta}\right]}{\left(l_{t}+R^{\alpha} s\right)\left(l_{t}+R^{\beta} s\right)}
\end{aligned}
$$

where $\alpha, \beta=A$ or $B$ and $\Omega$ is the atomic area. In this case the velocities of the two types of steps satisfy $v_{0}^{B}=-v_{0}^{A}$. Therefore the initial uniform step array is not a steady state. Depending on the direction of the electric field, one reconstruction domain expands while the other shrinks, creating step pairs separated by the minor terrace. With a downhill current one finds double height $D_{B}$ steps (consisting of an upper $S_{B}$ step and a lower $S_{A}$ step with a narrow $A$ terrace trapped in between) separated by wide $B$ terraces; the equivalent configuration 


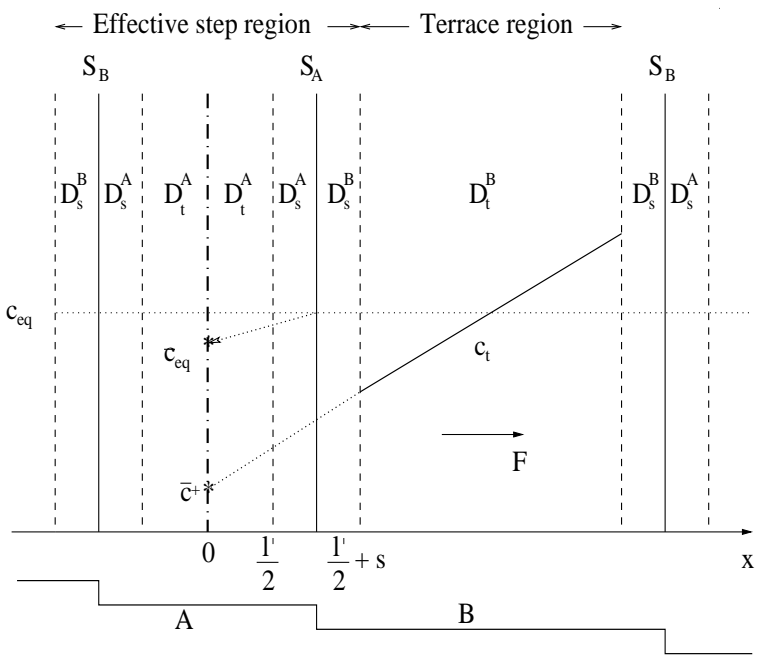

FIG. 4: A schematic illustration of extrapolation for an effective step region. With a step-down current, domain $(1 \times 2)$ expands to form an effective terrace region with some typical concentration profile $c_{t}$. On the other hand, domain $(2 \times 1)$ shrinks to $l^{\prime}$ and forms an effective step region when combined with the two step regions bounding it. $c_{t}$ is extrapolated to the dotted-dashed line at $x=0$ in the middle of the minor terrace which represents the effective "sharp" step.

with $D_{A}$ steps and narrow $B$ terraces is seen for an uphill current. Experiments show that this field-driven step pairing continues until it is balanced at short distances, probably by step repulsions, as first suggested by Natori et al. ${ }^{21}$ in the special case where local equilibrium was assumed for all the steps, corresponding to $R^{A}=R^{B}=1$ in our model.

\section{B. Continued Bunching of Paired Steps}

Now let us examine the stability of arrays of such paired steps. Assuming that the step pairs (boundaries of the minor domain) with constant spacings persist throughout the bunching process, as is shown by experiments, we can define a symmetric effective two-region model that can describe the continued bunching of the paired steps. To that end, we treat the minor reconstruction terrace together with the two step regions bounding it as an effective step region that separates one major terrace from another, as schematically shown in Fig. 4 for the case of a step-down current. As shown below (and previously discussed ${ }^{7}$ ), the bunching behavior is determined by the field direction and the sign of the kinetic coefficient for the sharp step model associated with the effective two-region model defined here.

In the Appendix we discuss the mapping to a sharp step model from a 1D hopping model that treats the effects of the electric field explicitly, while assuming there is a perfect sink at $x=0$ in the center of the step region. In the present case a minor terrace resides at the cen- ter of the effective step region. We can still follow basic treatment in the Appendix if we take this into account by shifting the origin of the coordinate system by transforming $x \rightarrow x-\left(l^{\prime}+s\right) / 2$ and $s / 2 \rightarrow l^{\prime} / 2+s$, where $l^{\prime}$ is the width of the minor reconstruction domain. Representing the discrete terrace concentrations by a continuum function $c(x)$ and Taylor expanding as before about $x=0^{+}$ - the center of the effective step region - we find that the effective sharp interface boundary condition takes a form analogous to Eq. A5:

$$
D_{t}^{\alpha}\left[\left.\nabla c\right|_{+}-f \bar{c}^{+}\right]=\bar{k}^{\alpha}\left(\bar{c}^{+}-\bar{c}_{e q}\right),
$$

where $D_{t}^{\alpha}, \alpha=A$ or $B$, is the normal diffusion constant in the major terrace. The continuum profile is schematically depicted in Fig. 团 for the case of a step-down current.

Two new features are seen in Eq. (23) arising from the use of a single effective step region to describe the paired steps. First, the major terrace is determined by the current direction in the initial step pairing regime. Second, both an effective kinetic coefficient $\bar{k}$ and an effective "equilibrium concentration" $\bar{c}_{e q}$ appear in the sharp step boundary condition. The latter is given by

$$
\bar{c}_{e q}=c_{e q}\left[1-\frac{1}{2} f\left(l^{\prime}+s\right)\right] .
$$

This can be understood heuristically by noting that the effective density in the center $\bar{c}_{e q}$ should be linearly modified by the weak field from its value $c_{e q}$ at the "real" local equilibrium step near the lower boundary of the effective step region. Similarly, the effective attachment length $\bar{d}^{\alpha}$ associated with the effective kinetic coefficient $\bar{k}^{\alpha}$ is given by

$$
\bar{d}^{\alpha} \equiv \frac{D_{t}^{\alpha}}{\bar{k}^{\alpha}}=\frac{\bar{s}}{2}\left[\bar{R}^{\alpha}-1\right],
$$

where $\bar{s}=l^{\prime}+2 s$ is the width of the effective step region and $\bar{R}^{\alpha}=s R^{\alpha} / \bar{s}$ is the relative diffusivity in the effective two region model defined above.

Equations (23)- 251) give the mapping between the effective two region model describing paired steps separated by major terraces and an equivalent sharp step model. In the steady state where the major terraces all have the same width, the surface flux in the sharp step model can be obtained from Eq. (21) as follows. We replace the parameters $c_{e q}, s$, and $R^{\alpha}$ by the corresponding effective parameters $\bar{c}_{e q}, \bar{s}$, and $\bar{R}^{\alpha}$. Clearly $l=l_{t}+\bar{s}$ represents the terrace width in the sharp step model. The steady state flux in the sharp step model as a function of the terrace width is thus given by

$$
J_{0}^{\alpha}(l)=D_{t}^{\alpha} c_{e q} f \frac{l}{l+2 \bar{d}^{\alpha}} .
$$

Note that $\alpha=A$ or $B$ is determined by the current direction.

To examine the stability of the above steady state, consider a small deviation $\delta x_{n}=\varepsilon_{n} e^{\omega t}$ of the $n^{t h}$ step 
in the uniform step train, where $\varepsilon_{n}=\varepsilon e^{i n \phi}$. Here $\varepsilon$ is a small constant and $\phi$ is the phase between neighboring steps. Then the $n^{\text {th }}$ step will move in response to the unbalanced flux induced by the changed widths of the terraces in front $l_{n}=l+\varepsilon_{n}\left(e^{i \phi}-1\right)$ and back $l_{n-1}=l+\varepsilon_{n}\left(1-e^{-i \phi}\right)$. The linear amplification rate $\omega=v_{n} / \varepsilon_{n}$ is given by

$$
\omega=\Omega D_{t}^{\alpha} c_{e q} \frac{4 \bar{d}^{\alpha} f}{\left(l+2 \bar{d}^{\alpha}\right)^{2}}(1-\cos \phi) .
$$

An instability towards step bunching results if $\bar{d}^{\alpha} f>0$ with a maximum at $\phi=\pi$, corresponding to step pairing. Note that the direction of the field and the sign of the effective kinetic coefficient combine to determine when step bunching occurs, as discussed earlier $\underline{7}$

Using Eqs. 25) and (27), we see that to get simultaneous step bunching from current in both directions, as seen in experiment, requires

$$
R^{A}>2+\frac{l^{\prime}}{s}>R^{B}
$$

With a step-down current, the first part of the inequality in Eq. (28) makes the effective kinetic coefficient for the effective step region containing the slower diffusion domain positive, which results in a step bunching instability. The second inequality in Eq. (28) give rise to a negative effective kinetic coefficient which produces step bunching with a step-up current. Note that this does not require negative kinetic coefficients for single steps of either kind.

However, if one assumes the individual steps are at local equilibrium, $\left(R^{A}=R^{B}=1\right)$, then the kinetic coefficient for the effective step region is negative in both cases, and therefore bunching is expected only from a step-up current.

\section{Angular Dependence}

It is straightforward to extend the above analysis to a general dimple geometry, where the domain conversion exhibits interesting angular dependences. A schematic view of the experimental dimple is shown in Fig. Ba. Again, we need to consider the fluxes from the neighboring terraces going into the step. Using Eq. (21), we can represent the surface flux as the sum of fluxes along the two characteristic directions,

$$
\mathbf{J}_{f}=\cos \theta J_{0}^{B} \widehat{\|}+\sin \theta J_{0}^{A} \widehat{\perp}
$$

for the front terrace and

$$
\mathbf{J}_{b}=\cos \theta J_{0}^{A} \widehat{\perp}+\sin \theta J_{0}^{B} \widehat{\|}
$$

for the back terrace of step 1 in Fig. 3k, where $\|$ and $\perp$ are the directions parallel and perpendicular to dimer rows as defined earlier. The angular dependent step velocity is readily obtained

$$
v_{0}^{(1)}(\theta, \varphi)=v_{0}^{B} \cos (2 \theta-\varphi),
$$

where $v_{0}^{B}$ is given by Eq. (22). Eq. (31) shows that a steady state of paired steps will form on the part of the dimple where $\cos (2 \theta-\varphi) \neq 0$.

In the following, we will concentrate on two special configurations that are studied experimentally $\underline{\underline{16}}$ The first is shown in Fig. 3 $\mathrm{b}$, where the current is parallel to the dimer row direction. In this case $\theta=0$, and $\cos \varphi$ characterizes the angular dependence around the dimple. The maximum pairing instability occurs at $\varphi=0$ where the current is perpendicular to the step normal direction, and no instability in seen at $\varphi= \pm \pi / 2$. From the previous discussion in Sec IVA and Sec IVB we can easily see that continued step bunching occurs with a maximum at $\varphi=0$.

The other interesting configuration corresponds to an upright field parallel to [010] direction in Fig. 3 $\mathrm{k}$. In this case the current is at an angle $\theta=\pi / 4$ from the dimer row direction. Hence the angular dependence becomes $\cos (\pi / 2-\varphi)=\sin \varphi$. The maximum pairing instability occurs at $\varphi=\pi / 2$, where the current is parallel to the steps, and no instability occurs when the current is perpendicular to the steps. Again the sharp step model corresponding to the steady state can be extracted. The subsequent step bunching instability for a parallel current was discussed by Liu et al ${ }^{22}$ Their stability analysis suggests that step bunching generally occurs for a nonvanishing attachment/detachment length $d$, regardless of its sign, when the current is parallel to the average step positions.

The results discussed here are in good agreement with experiments. For the angular dependent step pairing, the result is consistent with the original analysis by Nielsen et al. ${ }^{16}$ However, our explanation for the subsequent step bunching is different. Our analysis provide a simpler scenario that does not require a tensor character to the effective charge.

\section{CONCLUSION}

This paper derives expressions for sharp step boundary conditions characterized by linear kinetics rate parameters $k_{ \pm}$and $P$ for general BCF type models by appropriate coarse-graining from a microscopic hopping model. $k_{ \pm}$and $P$ are related to the attachment/detachment kinetics at kinks and to diffusion across the ledges respectively. In particular, our study shows that both parameters can be negative when diffusion is faster in the step region than on terraces. The possibility of negative kinetic coefficients was first suggested by Politi and Villain ${ }^{23}$ but with no derivation or discussion of any physical consequences. In the appropriate limit, we recover the mapping previously obtained with the CTRM $\stackrel{\underline{7}}{\underline{7}}$ Our results 
also seem consistent with those from phase field models,$\frac{9}{9}$ while providing a simple and physically suggestive picture.

We then used the perfect sink limit $\left(p_{k}=1\right)$ of the general model to analyze current-induced instabilities on $\operatorname{Si}(001)$, where the field represents the only driving force away from equilibrium, and found results in good agreement with experiment. As we will show elsewhere, this same limit also provides a coherent interpretation of $\mathrm{Si}(111)$ electromigration experiments, where novel step wandering behavior is seen ${ }^{17}$ Thus we believe that the perfect sink model provides a simple and physically reasonable description of many electromigration experiments on vicinal Si surfaces.

\section{Acknowledgments}

We are grateful to Ted Einstein, Oliver Pierre-Louis, and Ellen Williams for stimulating discussions. This work has been supported by the NSF-MRSEC at the University of Maryland under Grant No. DMR 00-80008.

\section{APPENDIX A: 1D PERFECT SINK MODEL WITH A CONSTANT ELECTRIC FIELD}

In the generic hopping model discussed earlier, we assumed that the flux arose only from concentration gra- dients. We consider here the case where there is a additional external driving force from the electric field, and take the perfect sink limit $p_{k}=1$ used to analyze experiments. In particular, we examine whether or not the kinetic rate parameters in the resulting sharp step model could depend on the field as Suga et al. previously suggested ${ }^{24}$

In the absence of the field, the 1D potential energy surface is similar to that in Fig. 11 where now the site at $x=0$ is a perfect sink surrounded by a more general region of width $s$ with different diffusion barriers. When a weak electric field is applied in the positive $x$ direction, the potential energy surface will be modified by an amount $V=-\int \mathbf{F} d \mathbf{x}$, where $\mathbf{F}=z^{*} e \mathbf{E}, z^{*} e$ is the effective charge. The modification of the potential surface produces a bias for adatom hopping, which will later lead to a convective flux contribution in the continuum description.

The driven flux inside the step region can be written as

$$
J_{x+a / 2}=\frac{D_{s}}{a} e^{f a / 2} \hat{c}_{s}(x)-\frac{D_{s}}{a} e^{-f a / 2} \hat{c}_{s}(x+a),
$$

where $f \equiv|\mathbf{F}| / k_{B} T$. The quasi-static approximation suggests continuity of fluxes, i.e. $J_{x+a / 2}=J_{x-a / 2}$, which leads to the following equation for the discrete concentration $\hat{c}_{s}(x)$,

$$
0=e^{-f a / 2} \hat{c}_{s}(x+a)-\left(e^{f a / 2}+e^{-f a / 2}\right) \hat{c}_{s}(x)+e^{f a / 2} \hat{c}_{s}(x-a),
$$

where $x$ is evaluated at discrete lattice sites inside the step region. It is easy to write down the solution of Eq. (A2) as

$$
\hat{c}_{s}(x)=c_{e q}+A\left(e^{f x}-1\right)
$$

taking account of the perfect sink at $x=0$. Here $A$ is a constant that can be determined by continuity of fluxes at the boundary between step and terrace region, i.e. $J_{s / 2-a / 2}=J_{s / 2+a / 2}$. This gives

$$
A=\frac{e^{-f a / 2} R \hat{c}_{t}\left(\frac{s}{2}+a\right)-\left[e^{-f a / 2}+e^{f a / 2}(R-1)\right] c_{e q}}{e^{f a / 2}\left(e^{f s / 2}-1\right) R+e^{f a / 2}-e^{-f a / 2}} .
$$

Here $\hat{c}_{t}$ is the discrete concentration on the terrace site.

To obtain the sharp interface boundary condition, we apply flux continuity $J_{s / 2+a / 2}=J_{s / 2+3 a / 2}$, and express all the discrete terrace concentrations in terms of the extrapolated $c^{+}$and the corresponding gradient $\left.\nabla c\right|_{+}$. In the weak field limit that is valid in most experiments, we can linearize the exponentials in all of the above expressions. To the leading order, we obtain the boundary condition as

$$
\pm D_{t}\left[\left.\nabla c\right|_{ \pm} \mp f c^{ \pm}\right]=k_{ \pm}\left(c^{ \pm}-c_{e q}\right)
$$

where results for both the + and - sides can be given by symmetry. Note that the term proportional to $f$ is the convective flux induced by the field, which is of the same order as the concentration gradient. As mentioned earlier in Section III the mapping to the kinetic coefficient is independent of the field to lowest order, and is given by

$$
d_{ \pm} \equiv \frac{D_{t}}{k_{ \pm}}=\frac{1}{2}\left(R_{ \pm}-1\right) s
$$

where $R_{ \pm} \equiv D_{t} / D_{s}$. Equation (A6) recovers the results we derived earlier from CTRM, and is also consistent with the general result in Eq. (13). 
* Present and permanent address: KLA-Tencor Corporation, Hatikshoret St, Migdal Haemek, Israel

1 A recent review of vicinal surfaces and step dynamics can be found in H.-C. Jeong and E. D. Williams, Surf. Sci. Repts. 34, 171 (1999), and references therein.

2 W. K. Burton, N. Cabrera, and F. C. Frank, Proc. R. Soc. London, A 243, 299 (1951).

3 A. A. Chernov, Sov. Phys. Crystallog. 1, 88 (1956).

4 A. A. Chernov, Sov. Phys. Uspekhi. 4, 116 (1961).

${ }^{5}$ R. L. Schwoebel, J. Appl. Phys. 40, 614 (1969).

6 M. Ozdemir and A. Zangwill, Phys. Rev. B 45, 3718 (1992)

${ }^{7}$ T. Zhao, J. D. Weeks, and D. Kandel, (preprint)

${ }^{8}$ R. Ghez and S. S. Iyer, IBM J. Res. Develop. 32, 804 (1988).

${ }^{9}$ O. Pierre-Louis, Phys. Rev. E 68, 021604 (2003).

10 O. Pierre-Louis and J. J. Métois (preprint)

11 For a comprehensive review see K. Yagi, H. Minoda, and M. Degawa, Surf. Sci. Repts. 43, 45 (2001) and references therein.

12 A. V. Latyshev, A. L. Aseev, A. B. Krasilnikov, and S. I. Stenin, Surf. Sci. 213, 157 (1989).

13 M. Degawa, K. Thürmer, I. Morishima, et al., Surf. Sci.
487, 171 (2001).

14 T. Doi, M. Ichikawa, S. Hosoki, Phys. Rev. B 55, 1864 (1997).

15 A. V. Latyshev, L. V. Litvin, A. L. Aseev, Appl. Surf. Sci. 130-132, 139 (1998).

16 J.-F. Nielsen, M. S. Pettersen, and J. P. Pelz, Surf. Sci. 480, 84 (2001).

17 T. Zhao, J. D. Weeks, and D. Kandel, unpublished.

18 For a general review see H. J. W. Zandvliet, Rev. Mod. Phys. 72, 593 (2000) and references therein.

19 Y. W. Mo and M. G. Lagally, Surf. Sci. 248, 313 (1991).

${ }^{20}$ We use the notation of J. Myslivecek, C. Schelling, F. Schaffler, et al., Surf. Sci. 520, 193 (2002), who examine related instabilities during growth on $\mathrm{Si}(001)$.

21 A. Natori, H. Fujimura, and H. Yasunaga, Jpn. J. Appl. Phys. 31, 1164 (1992)

22 D.-J. Liu, J. D. Weeks, and D. Kandel, Phys. Rev. Lett. 81, 2743 (1998).

23 P. Politi and J. Villain, Phys. Rev. B 54, 5114 (1996).

24 N. Suga, J. Kimpara, N.-J. Wu, H. Yasunaga, and A. Natori, Jpn. J. Appl. Phys. 39, 4412 (2000). 